\title{
Systems approaches to risk assessing healthcare, how far have we come?
}

\author{
Peter Buckle \\ Robens Institute, Guildford, England and Visiting Professor, University of Nottingham.
}

\begin{abstract}
Systems approaches and their relevance to risk assessment are considered through an examination of a number of recent applications in England. The importance of visual mapping of systems is emphasised. This approach encourages participation from those within the system with less technical knowledge but with important information to add to a risk assessment. Other advantages outlined include the ability to readily identify system boundaries, map processes and record both strengths and weaknesses of existing or planned system changes. The use of risk assessment methods with this approach should be cautious, as any one method may only identify a sub-set of all risks.
\end{abstract}

Keywords: Systems analysis, healthcare, mapping, risk

\section{Introduction}

This paper considers the extent to which systems approaches to healthcare and risk assessment have developed in the UK in recent years. The complexity of modern healthcare has resulted in the need for robust systematic approaches to design of care delivery [6], yet all too often these appear to be limited to perspectives that exclude the essence of good ergonomics, i.e. user centric approaches. The illusion of 'patient focused' approaches often appears to ignore the needs of those must deliver the care. Innovations in health care abound, as does exemplars of technology that failed or was discarded because human factors were not considered at the outset.

\section{Systems Mapping}

Some researchers have attempted to address this through a fuller consideration of the components of the system prior to commencing risk assessments and interventions.

We have previously reported [4] a study that mapped the critical systems components involved in the delivery of pharmaceutical products in healthcare.
Systems maps (see figure 1) were generated with the help of those professionals responsible for delivering and maintaining the system.

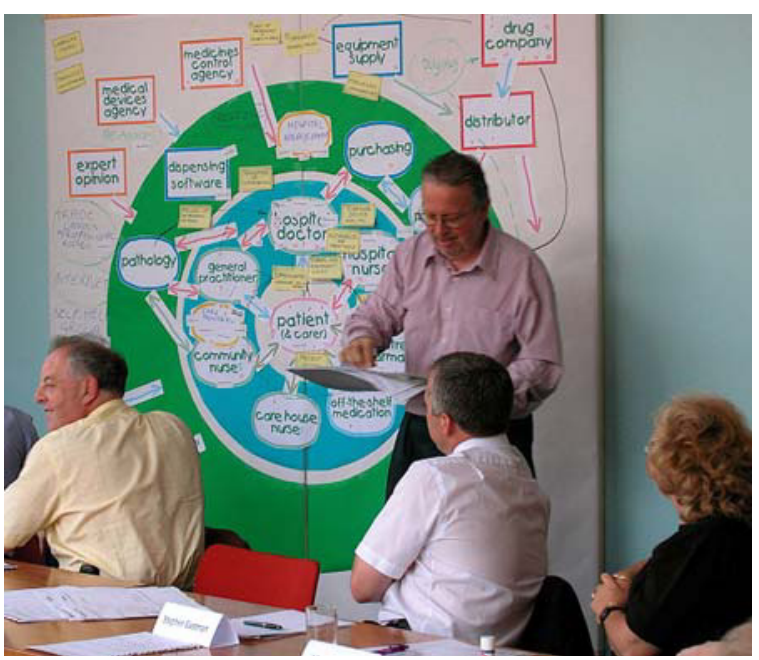

Figure 1 Systems map generation

The final map [7] illustrates the complexity of the system. This complexity may not be surprising when the range of pharmaceutical products available in modern healthcare is taken into account. However, a more recent study [2,3] demonstrates that for even a 'simple' technological test in healthcare (i.e. nasoga- 
strictube placement) the system involves a sizeable set of people, equipments and environments.

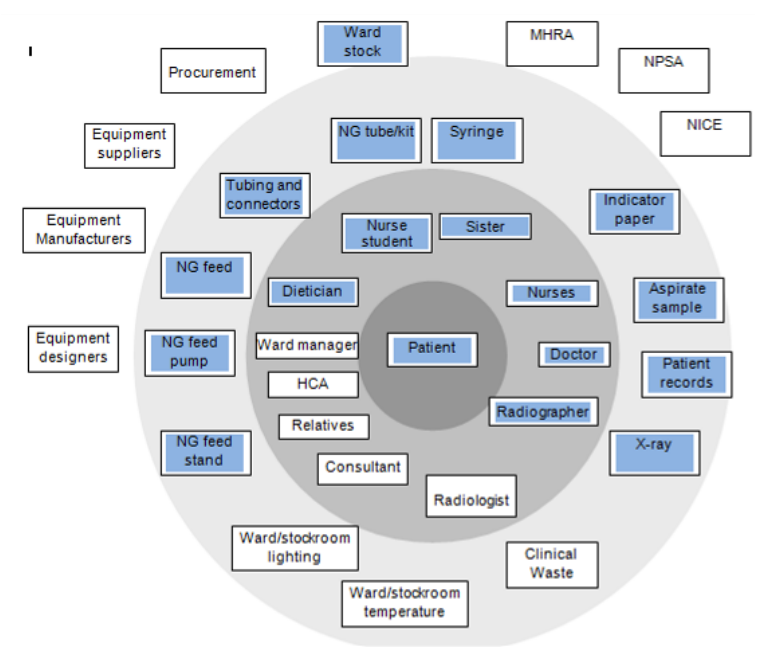

Figure 2 Nasogastric tube placement: systems map

System descriptions may also suffice in this regard. However, in the context of validation we have generally found that all levels of professional healthcare givers and patients and their representatives can identify with the mapping process [4]. Such an approach has the added advantage of helping identify variations in processes, locations and those with responsibility for task delivery.

\section{Systems Maps and Risk Assessment}

Risk assessment in healthcare is generally perceived as being complex because of the innate variability of patients, their conditions and professional practices. The use of systems maps enables a number of important considerations to be readily addressed.

i. The components of the system being risk assessed can be readily identified. These components include, for example, people, objects and environments.

ii. All those who have a valid understanding of these elements can be invited to contribute to the risk assessment process. In a recent study of nasogastric tube placement this ensured that, not only were those that administered the positional 'test' involved, but also others who had a responsibility for monitoring the patient and checking the tube position. Each member of the risk assessment group contri- buted valuable information that helped elucidate the advantages and disadvantages of the existing system.

iii. The map can be used to clarify processes occurring in health care. It is relatively simple to document on a systems map, through a system of line drawings, that show how and where processes are occurring. Jun et al [8] have provided an important set of methods that show how processes may be documented in health care.

iv. The use of the map in this manner demonstrates very clearly which aspects of the system are being assessed and which are not. This aids in the understanding and recognition of where the system boundaries are being set. With this knowledge it is then relatively straightforward to ensure that the resultant . risk assessment (or potential impact of an intervention in healthcare) remain realistic and pragmatic in their scope $[5,7]$.

v. Those with important perspectives of risk within systems may be bemused or excluded by the complexity of language that accompanies many such exercises. Language that is appropriate for systems engineers or trained medical staff may seem alien to patients and their carers. Yet all such users and contributors to the system may bring valid knowledge and experience to the risk assessment exercise. We have found [4] that the simple use of post-it notes and a large map of the system encourages all users to contribute to the identification of both strengths and weaknesses of the existing system. Such an approach can also be used with any intended changes to the system.

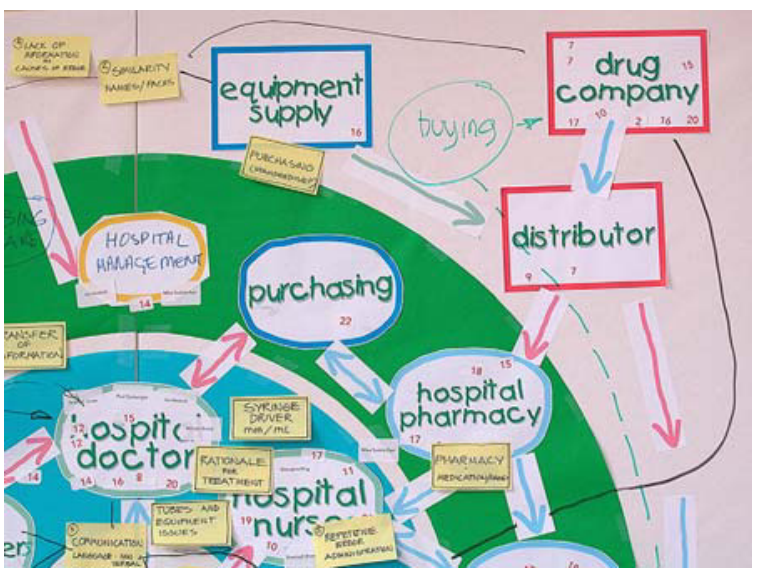

Figure 3 Detail of medication delivery system 


\section{Risk assessment methods}

Researchers [9] have refined and identified a set of risk assessment methods suitable for use in healthcare. This invaluable toolkit clarifies and simplifies the effective use of such methods. As such it is an important resource. Whilst aimed at users across the healthcare system with some prior knowledge of risk assessment, it's approach is simple and can be appreciated by all. By placing a strong emphasis on mapping processes prior to assessment it helps fulfill one additional by product of such methods, namely a fuller understanding of the system and interactions between components. In the view of this author, this activity alone is essential for identifying opportunities to improve healthcare safety and quality.

Use of any single risk assessment method may be fraught with dangers. A recent paper [1] demonstrates this clearly. Use of Failure modes and effects analysis (FMEA) and 'So what if?' techniques (SWIFT) produced substantially different outcomes when applied to a community-based anticoagulation and stroke prevention service. Whilst there was some degree of congruence, each method clearly identified a set of potential risks not identified by the other.

The sheer volume of failure modes identified in many healthcare analyses also means that other avenues (other than removal of the risk) may need to be explored. We have found it helpful to consider how resilience forms part of this picture. It would seem important to establish how mapping and risk assessment might be supplemented with resilience mapping to strengthen health care processes. Such an approach would lend itself to designing better failure/disaster recovery programmes for those in a position to act.

\section{Conclusions}

Systems approaches to understanding healthcare are not new. However, improved participation in risk assessment involving representatives of all appropriate user groups can be greatly enhanced using appropriate mapping. Other advantages of this approach are the clarity that it brings to the recognition of processes, those system elements involved in the process and the boundaries of the risk assessment exercise. The use of this approach in prospective risk assessment (e.g. when changes are contemplated) is regarded as essential. Current risk assessment methods are appropriate but careful selection is required and more than one method may be needed for any specific issue.

\section{References}

[1] Anderson, J et al (2011) Developing Prospective Hazard Analysis in Healthcare: Does it matter which method is used? Comparison of FMEA and SWIFT. Proceedings of HEPS 2011, Orviedo, Spain.

[2] Anderson, O Buckle, P and Hanna G (2012) Ergonomics risk assessment of nasogastric tube placement and implications for design and training Proceedings of the IEA Congress, Recife, Brazil

[3] Anderson, $\mathrm{O}$ et al Design, development and validation of a safer enzyme-based nasogastric tube position test Gastroenterology (under submission)

[4] Buckle, P Clarkson PJ, Coleman R, Bound, J, Ward J and Brown, J (2010) Systems mapping workshops and their role in understanding medication errors in healthcare Applied Ergonomics 41, 645-656

[5] Buckle P, Clarkson PJ, Coleman R, Ward J and Anderson J (2006) Patient safety, systems design and ergonomics Applied Ergonomics, 37, 491-500.

[6] Carayon, P et al (2006)Work system design for patient safety: the SEIPS model Qual Saf Health Care 15 5058

[7] Clarkson PJ, Buckle P, Coleman R, Stubbs D, Ward J, Jarrett J, Lane R, and Bound J (2004) Design for patient safety: a review of the effectiveness of design in the UK Health Service. Journal Engineering Design, $15,123-140$

[8] Jun et al (2009) Health care process modelling: which method when? Int J Qual Health Care) 21, 214-224

[9] Ward, J.R., Clarkson, P.J., Buckle, P., Berman, J., Lim, R. and Jun, G.T. (2010) 'Prospective hazard analysis: Tailoring prospective methods to a healthcare context', Patient Safety Research Portfolio, Report 